Contract No. and Disclaimer:

This manuscript has been authored by Savannah River Nuclear Solutions, LLC under Contract No. DE-AC09-08SR22470 with the U.S. Department of Energy. The United States Government retains and the publisher, by accepting this article for publication, acknowledges that the United States Government retains a non-exclusive, paid-up, irrevocable, worldwide license to publish or reproduce the published form of this work, or allow others to do so, for United States Government purposes. 


\title{
Patchy Silica-Coated Silver Nanowires as SERS Substrates
}

\author{
Simona E. Hunyadi Murph ${ }^{1}$ and Catherine J. Murphy ${ }^{2}$ \\ ${ }^{1}$ Savannah River National Laboratory \\ Savannah River Site, Aiken, SC 29808 \\ ${ }^{2}$ University of Illinois at Urbana-Champaign \\ Department of Chemistry \\ 600 S. Mathews Ave., Urbana IL 61801
}

*Corresponding author, email address: Simona.Murph@srnl.doe.gov

Phone: 1-803-646-6761, Fax: 1-803-725-4478

\begin{abstract}
We report a class of core-shell nanomaterials that can be used as efficient surfaceenhancement Raman scattering (SERS) substrates. The core consists of silver nanowires, prepared through a chemical reduction process, that are used to capture 4mercaptobenzoic acid (4-MBA), a model analyte. The shell was prepared through a modified Stöber method and consists of patchy or full silica coats. The formation of silica coats was monitored via transmission electron microscopy, UV-visible spectroscopy and phase-analysis light scattering for measuring effective surface charge. Surprisingly, the patchy silica coated silver nanowires are better SERS substrate than silver nanowires; nanomolar concentration of 4-MBA can be detected. In addition, "nano-matryoshka" configurations were used to quantitate/explore the effect of the electromagnetic field at the tips of the nanowire ("hot spots") in the Raman scattering experiment.
\end{abstract}




\section{Introduction}

In the last three decades, a great deal of interest has been directed toward the development of active substrates for surface enhanced Raman scattering (SERS) experiments (Jun et al 2006; Lisiecki et al 2005; Hunyadi Murph et al 2011, 2012; Puntes et al 2001; Scott et al 2005; Song et al 2012; Wiley et al 2005; Xia et al 2003; Yin et al 2005). It has been already reported that SERS can be used for detection of traces amount of substances for a large variety of molecules of environmental (Ruan et al 2006; Zhang et al 2006), biomedical (Kneipp et al 1999; Liu at al 2005; Vo-Dinh et al 2006), and pharmaceutical interest (Kneipp et al 1999; Pinzaru et al 2004). Widely used are silver and gold colloids which allow chemical detection of analytes at very low concentration with the potential for single molecule detection (Doering at al 2002; Kneipp et al 1997).

Today is generally accepted that the enhancement effects observed in the SERS are the results of two different effects: the electromagnetic (EM) effect, enhancement of the local electromagnetic field incident on an adsorbed molecule at a metallic surface; and the chemical effect; due to the electronic resonance/charge transfer between a molecule and a metal surface, which leads to an increase in the polarizability of the molecule (Orendorff et al 2005). While various nanoparticle shapes, such as spheres, cubes, triangles, etc. have been successfully employed as SERS substrate (dos Santos et al 2005, Jensen et al 1999; McLellan et al 2006; Orendorff et al 2006) one-dimensional nanostructures are extremely interesting as well (Hunyadi et al 2006; Nikoobakht et al 2003; Orendorff et al 2006; Wang et al 2005). Nanorods and nanowires when used as SERS substrate have the potential to produce an increased electric field at the tips of the particles. Tip enhanced Raman scattering (TERS) (Hartschuh et al 2003; Hayazawa et al 2001; Stöckle et al 2000), increase SERS signals (Imura et al 2004; Kelly et al 2003; Murphy et al 2005). TERS has been largely studied due to the fact that apex of metallic tip behaves as a nano-antenna and can be considered as a local electromagnetic source (Billot et al 2006). Moreover, the assembly of nanorods end-to-end in one-dimension, may be a good strategy to create SERS substrates; EM enhancement has been observed and attributed to nanowire plasmon coupling or SERS "hot spots" for molecules adsorbed in the fractal space between wires (Blatchford et al 1982; Creighton et al 1979Imura et al 
2004; Kelly et al 2003; Kerker et al 1984; Orendorff et al 2006; Murphy et al 2005; Solecka-Cermakova et al 1996).

The ability to use SERS in routine analyses requires the presence of stable, highly reproducible, inexpensive, and easily prepared substrates. The SERS enhancement effects are highly depended on the substrate preparation (Park et al 2006). By varying the nanoparticle dimensions, aggregation state, spacing, as well as the dielectric properties of the surrounding medium, it is possible to tune plasmon resonances, which can greatly enhance the sensing capabilities (Imura et al 2004; Jensen et al 1999; Hunyadi Murph et al 2011; Kelly et al 2003; Murphy et al 2005).

In this chapter, we describe a wet chemical approach to develop novel engineered one-dimensional nanostructures, with distinctive configuration, that can be used as effective SERS substrates. Silver nanowires were fully and partially functionalized with silica (Scheme 1, substrate B and C), by using a modification of the procedure reported earlier by us (Hunyadi et al 2006). This procedure is an indirect Stöber approach, and requires the use of 3-mercaptopropyltrimethoxysilane (MPTMS) as "molecular glue" for the silica coating, followed by the addition of sodium silicate. For SERS, the ananlyte of interest, 4-mercaptobenzoic acid (4-MBA), was captured onto silver nanowires by silica coats. The results of this approach produced SERS active silver nanowires (Scheme 1, substrate $\mathrm{B}$ and $\mathrm{C}$ ) that can detect nanomolar concentrations of the analyte of interest.

In the same time, through an ammonia treatment we produced "nano-matryoshka" configurations (Scheme 1, substrate D), consisting of concentric nanowires/nanotubes of metal/insulator nanomaterials. The metallic inner nanoparticle is made of an undersized (as a result of the reaction between $\mathrm{Ag}$ and ammonia) Ag nanowires functionalized with 4-MBA while the outer shell, the insulator, is the original, now oversized silica shell. These "nano-matryoshka" configurations were used to quantitate/explore the effect of the electromagnetic field at the tips of the nanowire ("hot spots") in the Raman scattering experiment. The development of these novel silver-silica nano-architectures opens new possibilities in using SERS for spectroscopic labeling of various markers, molecular sensing, recognition, etc. 


\section{Experimental Section}

2.1. Materials and Instrumentation: Silver nitrate $\left(\mathrm{AgNO}_{3}, 99 \%\right)$, trisodium citrate (99\%), 4-mercaptobenzoic acid (4-MBA), 3-mercaptopropyltrimethoxysilane (MPTMS, $95 \%)$, sodium silicate solution $\left(27 \% \mathrm{SiO}_{2}\right.$ in water), and ammonia solution $\left(\mathrm{NH}_{3}, 25 \%\right.$, $14 \mathrm{~N}$ ) were all from Sigma-Aldrich and used as received. Ethyl alcohol, USP absolute-200 proof, was provided by AAPER Alcohol and Chemical Co. and was used as received. Deionized ultra filtered water from Fisher Chemicals was used throughout the experiments. All glassware was cleaned with aqua regia and thoroughly rinsed with deonized ultra filtered water. Transmission electron microscopy was performed with a Hitachi 8000 transmission electron microscope on copper grids operating at $200 \mathrm{kV}$. UVvis spectroscopy was performed on a Cary 500 Scan UV-vis-NIR spectrophotometer. Potential measurements were performed on a Brookhaven Zeta phase analysis lightscattering system (PALS) instrument.

Surface-enhanced Raman spectra were collected using a Detection Limits Solution 633 Raman system using a 633-nm helium neon laser with $25-\mathrm{mW}$ laser power at the sample. Spectra were corrected for background using Grams 32 software (Galactic).

2.2. Synthesis of Silver Nanowires. Silver nanowires were prepared by a method previously reported (Caswell et al 2003). Basically, two different solutions, A and B, were prepared in $250 \mathrm{~mL}$ conical flasks. First solution A, contained $100 \mathrm{~mL}$ deionized water, $1.5 \mu \mathrm{L}$ of $1 \mathrm{M}$ aqueous $\mathrm{NaOH}$ and $40 \mu \mathrm{L}$ of $0.1 \mathrm{M}$ aqueous $\mathrm{AgNO}_{3}$ was brought to a boil with rapid stirring for $\sim 5$ minutes, with a $50 \mathrm{~mL}$ beaker as a lid. After this solution came to a boil, $5 \mathrm{~mL}$ of aqueous $0.01 \mathrm{M}$ trisodium citrate was added to it. The second solution, containing $150 \mathrm{~mL}$ deionized water, $1.5 \mu \mathrm{L}$ of $1 \mathrm{M}$ aqueous $\mathrm{NaOH}$ and $20 \mu \mathrm{L}$ of $0.1 \mathrm{M}$ aqueous $\mathrm{AgNO}_{3}$ was, also, brought to a boil with rapid stirring, with a $50 \mathrm{~mL}$ beaker as a lid. At this point, both solutions were boiled and refluxed for 10 minutes and after that solution A was added to solution B. These combined solutions were boiled for 50-60 minutes with rapid stirring. The resulting silver nanowires were further purified by centrifugation at $7000 \mathrm{rpm}$ for 10 minutes and used for the engineered Ag-silica nanomaterials. 


\subsection{Synthesis of Silver Nanowire Functionalized with 4-MBA (Scheme 1, Substrate}

A). As a control experiment, $500 \mu \mathrm{L}$ of a $2 \mathrm{mM}$ aqueous solution of 4-MBA was added to a $1 \mathrm{~mL}$ aliquot of Ag nanowires. After 24 hours incubation time in 4-MBA, the samples were purified by centrifugation at $7000 \mathrm{rpm}$ for 10 minutes and redispersed in DI water.

\subsection{Capturing 4-MBA with Patchy Silica Coats onto Silver Nanowires (Scheme 1,} Substrate B). Different concentrations of 4-MBA solutions $(650,260,130,65,13,6.5$, $1.3,0.66,0.33,0.16,0.083,0.041,0.02,0.01$, and $0.005 \mu \mathrm{M})$ were added to $1 \mathrm{~mL}$ aliquots of Ag nanowires. The final volume of the aliquot solutions was $1.5 \mathrm{~mL}$, by adding extra ethyl alcohol solution for identical experimental conditions. The solutions were then stirred for 20 minutes to ensure that 4-MBA was bound to the silver nanowire. Next, without any purification, $20 \mu \mathrm{L}$ of $0.2 \mathrm{mM}$ MPTMS was added to the solution and stirred for another 20 minutes. Finally, $5 \mu \mathrm{L}$ of a $0.252 \mathrm{M}$ aqueous sodium silicate $\left(\mathrm{Na}_{2} \mathrm{O}\left(\mathrm{SiO}_{2}\right)_{3}\right)$ solution was added to the sample, and the stirring was continued for another 20 minutes. The coated nanoparticles were allowed to "rest" for either 1 hour or 24 hours at room temperature. After that, the samples were separated by the reaction medium by centrifugation $(7000 \mathrm{rpm}$ for $10 \mathrm{~min})$ and redispersed in deionized water $(0.5$ $\mathrm{mL})$.

\subsection{Capturing 4-MBA with Full Silica Coats onto Silver Nanowires (Scheme 1,}

Substrate C). The continuous silica shell deposit on the entire Ag nanowire was produced when $25 \mu \mathrm{L}$ of $0.5 \mathrm{mM}$ MPTMS solution was added to a $1 \mathrm{~mL}$ aliquot of silver nanowire solution functionalized with 4-MBA. The 4-MBA functionalized process took place as follow: $200 \mu \mathrm{L}$ of a $2 \mathrm{mM}$ ethanol solution of 4-MBA was added into a $1 \mathrm{~mL}$ aliquot of silver nanowire solution for 1 hour, with an initial 20 minute stirring time. After purification and centrifugation this solution was stirred for 20 minutes to ensure that MPTMS was bound to the silver nanowires. Then $5 \mu \mathrm{L}$ of a $0.252 \mathrm{M}$ aqueous solution of sodium silicate $\left(\mathrm{Na}_{2} \mathrm{O}\left(\mathrm{SiO}_{2}\right)_{3}\right)$ solution was added, and the stirring was continued for another 20 minutes. The solution was left overnight to allowed formation of a continuous layer of silica. Finally, the coated nanoparticles were separated from the 
reaction medium by centrifugation (7000 $\mathrm{rpm}$ for $10 \mathrm{~min}$ ), washing and redispersion into deionized water.

\subsection{Synthesis of "Nano-Matryoshka" Configuration and Increase of "Hot Spots"} through an Ammonia Treatment (Scheme 1, Substrate D). The as formed Ag-4MBA-

silica nanomaterials, synthesized as described above in $\mathrm{C}$, were immersed in an aqueous ammonia solution $(25 \%, 14 \mathrm{~N})$ for 1 day, with a 20 minute initial stirring time. The next day, ammonia was removed from the solution by centrifugation and the nanowires were redispersed into DI water $(7000 \mathrm{rpm}, 10$ minutes). The new nanomaterials consist of concentric undersized Ag nanowires encapsulated in the original, "oversized" silica shell ("Nano-Matryoshka" configuration).

2.7. Synthesis of Silver-Silica Core-Shell Nanostructures without 4-MBA. In a control experiment, in the absence of 4-MBA, $1 \mathrm{~mL}$ aliquot of silver nanowire solution was functionalized with $20 \mu \mathrm{L}$ of $0.2 \mathrm{mM}$ MPTMS, with a 20 minute stirring time. After 1 hour incubation time in MPTMS, $5 \mu \mathrm{L}$ of $0.252 \mathrm{M}$ aqueous sodium silicate $\mathrm{Na}_{2} \mathrm{O}\left(\mathrm{SiO}_{2}\right)_{3}$ was added to the sample and allowed to sit overnight to allow formation of a uniform silica shell. Next day, the samples were purified (7000 rpm for 10 minutes) and used for the characterization. The transmission electron micrograph showed that the silica shell was uniform and homogeneous, with a thickness of $14 \pm 4 \mathrm{~nm}$.

\section{Results}

\subsection{Characterization of Silver Nanowires Engineered with Patchy Silica Coats while} Capturing Analyte Molecules (Scheme 1, Substrate B).

By using modification of the work reported by us (Caswell 2003), 4-MBA was captured onto Ag nanowires, in suspension, with patchy or full silica deposits to examine their relative abilities for Raman scattering experiments. The analyte of interest, 4-MBA, was first added to the silver nanowire, followed by the addition of a silane coupling agent, MPTMS. We found that addition of sodium silicate produced patchy silica deposits on silver nanowire surface. These patchy silica deposits could be expanded to generate a full silica shell over silver nanowire core by increasing the reactant concentrations. 
The nanomaterials were characterized by transmission electron microscopy (TEM), zeta potential measurements, uv-visible absorption spectroscopy and Raman spectroscopy.
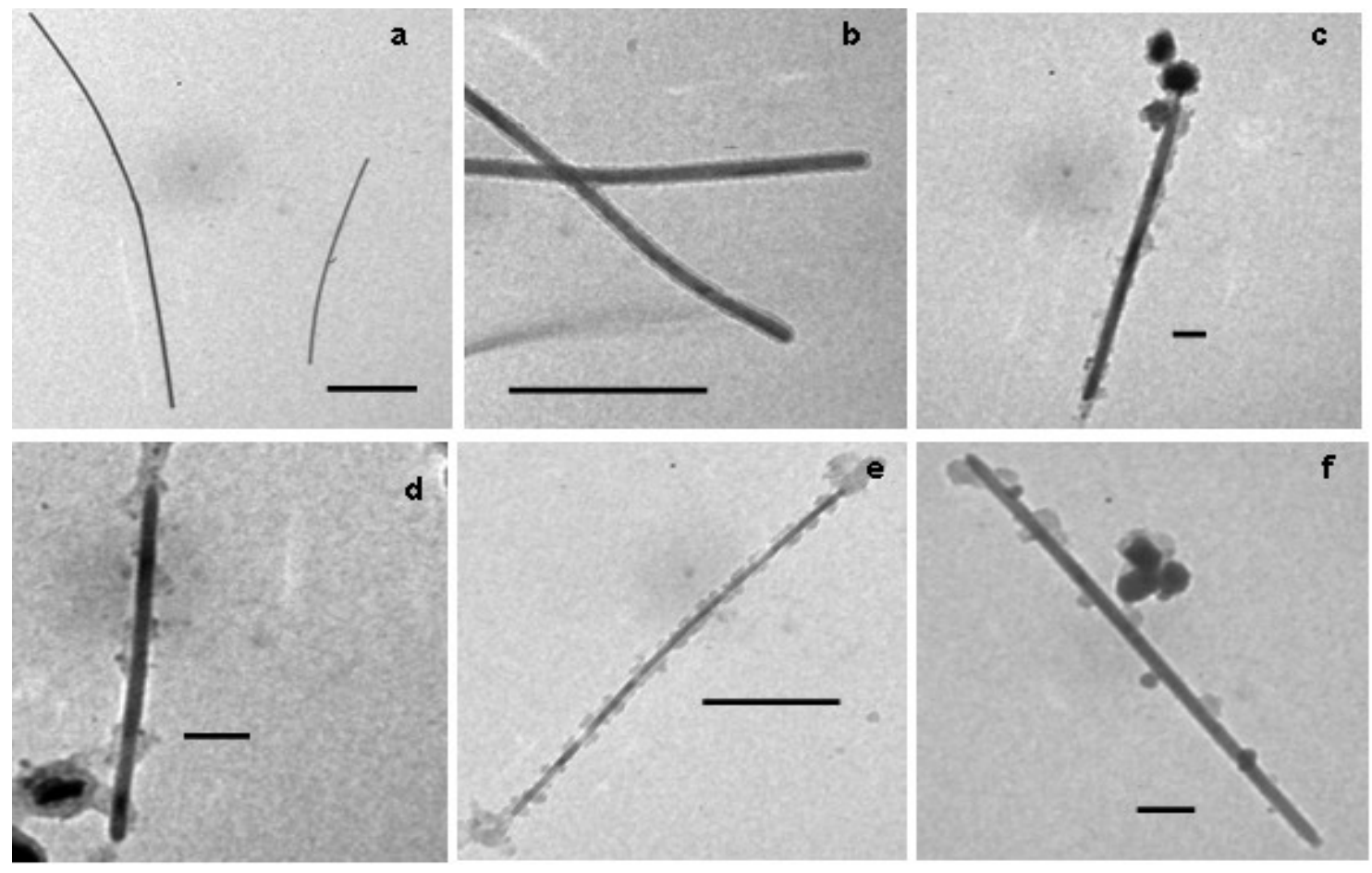

Figure 1. TEM images of (a) Ag nanowires, (b) Ag-silica core-shell nanowires, (c) Ag nanowires with a patchy silica coat, made in the presence of $0.08 \mu \mathrm{M} \mathrm{4-MBA;}$; d) Ag nanowires with a patchy silica coat, made in the presence of $0.66 \mu M$ 4-MBA; (e) capturing 6.5 $\mu M$ 4-MBA with patchy silica onto Ag nanowires, (f) Ag nanowires with a patchy silica coat, made in the presence of $650 \mu M$ 4-MBA. Scale bar: a-2microns, $b, e-$ $500 \mathrm{~nm}, c, d, f-100 \mathrm{~nm}$.

Silver nanowires (width $30 \pm 3 \mathrm{~nm}$, length from 200 up to 20 microns; Fig. 1a) were prepared as previously described. ${ }^{18}$ Without 4-MBA, full silica coating of variable thickness can be produced (a single example is shown in Figure 1b). The transmission electron micrograph images show that patchy silica deposits were formed onto $\mathrm{Ag}$ nanowires in the presence of 4-MBA (Figure 1c-f). In the case of patchy silica produced onto $\mathrm{Ag}$ nanowires, silica patches thicknesses fluctuated from area to area without any consistence or regularity. Moreover, the TEM images revealed that $\sim 60-80 \%$ of the wires had free ends without any silica coatings. The ends of the nanowires play an important role in the surface enhanced Raman scattering experiments, due to the fact that they can be considered "hot spots" which can produce an additional enhancement effect. 
Changes of the 4-MBA concentration (from $650 \mu \mathrm{M}$ to $0.005 \mu \mathrm{M}$ ) produced silica patches onto the silver surface with variable length from $27 \pm 2$ to $61 \pm 4 \mathrm{~nm}$.

In the absence of 4-MBA, $100 \%$ of the silver surface area was coated with silica. In this case, the thickness of the silica shell formed over the entire silver nanowire core surface was $14 \pm 1 \mathrm{~nm}$ (Figure 1b).

The UV-Vis absorption spectra were used to characterize the engineered nanomaterials. Before the silica deposition, the Ag nanowires present a transverse plasmon band at around $\sim 410 \mathrm{~nm}$. The longitudinal plasmon band is past $1400 \mathrm{~nm}$ (Hunyadi et al 2006). Upon deposition of patchy silica coats on the Ag nanowires, in the presence of 4-MBA, a red shift of the transverse plasmon band for silver nanowires, from $\sim 410 \mathrm{~nm}$ to $\sim 470 \mathrm{~nm}$ was observed (Figure $2 \mathrm{a}, \mathrm{b}$ ). These results are in agreement with data reported by others (Chen et al 2002; Feldheim 2002; Liz-Marzan et al 1996; Mine et al 2003; Obare et al 2001; Santra et al 2001), due to the silica high constant dielectric of $\varepsilon=4.5$ (Obare et al 2001).

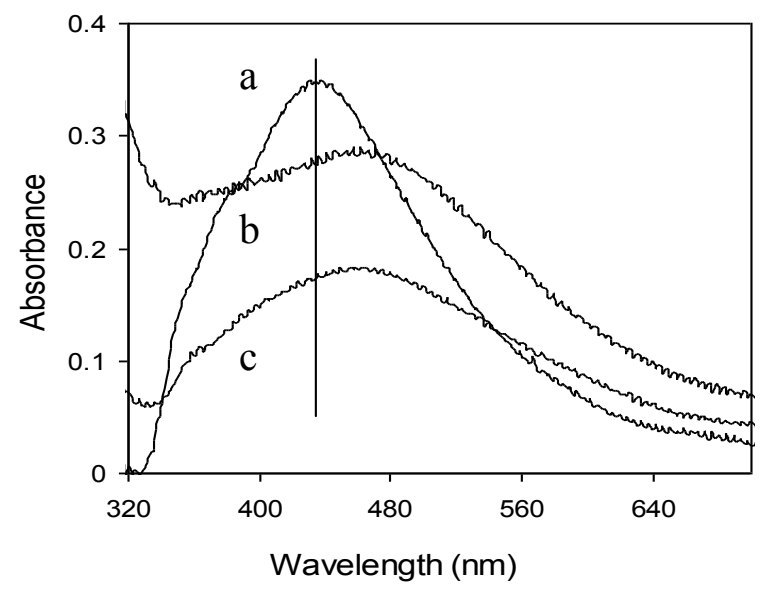

Figure 2. UV-Vis spectra of (a) Ag nanowires, (b) Ag nanowires coated with patchy silica coats, and (c) full core-shell Ag-silica nanowires.

Zeta potential measurements were performed on a phase analysis light-scattering system to monitor the surface charge during the experiments. As expected, when $\mathrm{Ag}$ nanowires were engineered with silica coats, their surface charge changed significantly. Initially, the Ag nanowire surface charge was $-24 \pm 0.9 \mathrm{mV}$, presumably as a result of the 
$\mathrm{OH}^{-}$adsorbed on the surface. After addition of the $500 \mu \mathrm{L} 0.1 \mathrm{mM}$ MBA onto a $1 \mathrm{~mL}$ aliquot of Ag nanowires, the surface charge became $-32 \pm 2 \mathrm{mV}$. This is probably due deprotonation of carboxylic acid. Patchy silica-coated silver nanowires, in the presence of $650 \mu \mathrm{M}$ of $4-\mathrm{MBA}$, showed a $-55.8 \pm 2 \mathrm{mV}$.

\subsection{Raman Detection of 4-MBA with Silver Nanowires Engineered with Patchy Silica Coats (Scheme 1, Substrate B).}

The surface enhanced Raman scattering (SERS) activity of silver nanowires functionalized with patchy silica coats, in the presence of 4-MBA, (Scheme 1, Substrate B) was examined at room temperature after $1 \mathrm{hr}$. and after $24 \mathrm{hrs}$. incubation time at various concentration of 4-MBA. The vibrational spectrum of 4-MBA was reported earlier (Hunyadi et al 2006; Orendorff et al 2005, 2006), and the characteristic peaks expected in our experiments were: $v(\mathrm{CC})_{\text {ring }}$ ring-breathing modes $\left(1070 \mathrm{~cm}^{-1}\right.$ and 1575

$\left.\mathrm{cm}^{-1}\right), \delta(\mathrm{CH})$ bends $\left(1132 \mathrm{~cm}^{-1}\right.$ and $\left.1173 \mathrm{~cm}^{-1}\right), v_{\mathrm{s}}\left(\mathrm{COO}^{-}\right)$stretches $\left(1357 \mathrm{~cm}^{-1}\right)$ (Hunyadi et al 2006; Orendorff et al 2005, 2006).

Figure 3 shows the Raman spectra of $650 \mu \mathrm{M} 4-\mathrm{MBA}$ in the presence of three substrates: "bare" Ag nanowires (Scheme 1, Substrate A), Ag nanowires functionalized with patchy silica (Scheme 1, Substrate B), and Ag nanowires fully coated with silica (Scheme 1, Substrate C). The vibration bands at $1070 \mathrm{~cm}^{-1}$ and $1575 \mathrm{~cm}^{-1}$ in 4-MBA, which are assigned to be the $\mathrm{v}(\mathrm{CC})_{\text {ring }}$ ring-breathing modes, were found to be greatly enhanced. No shifts were observed in the case of SERS spectra. Surprisingly, patchy coats of silica on silver nanowires produce by far the largest signal for 4-MBA. This suggest that the electromagnetic enhancement is associated with the substrate dielectric properties of the surrounding medium, which has been suggested by others (Imura et al 2004; Kelly et al 2003; Murphy et al 2005). 


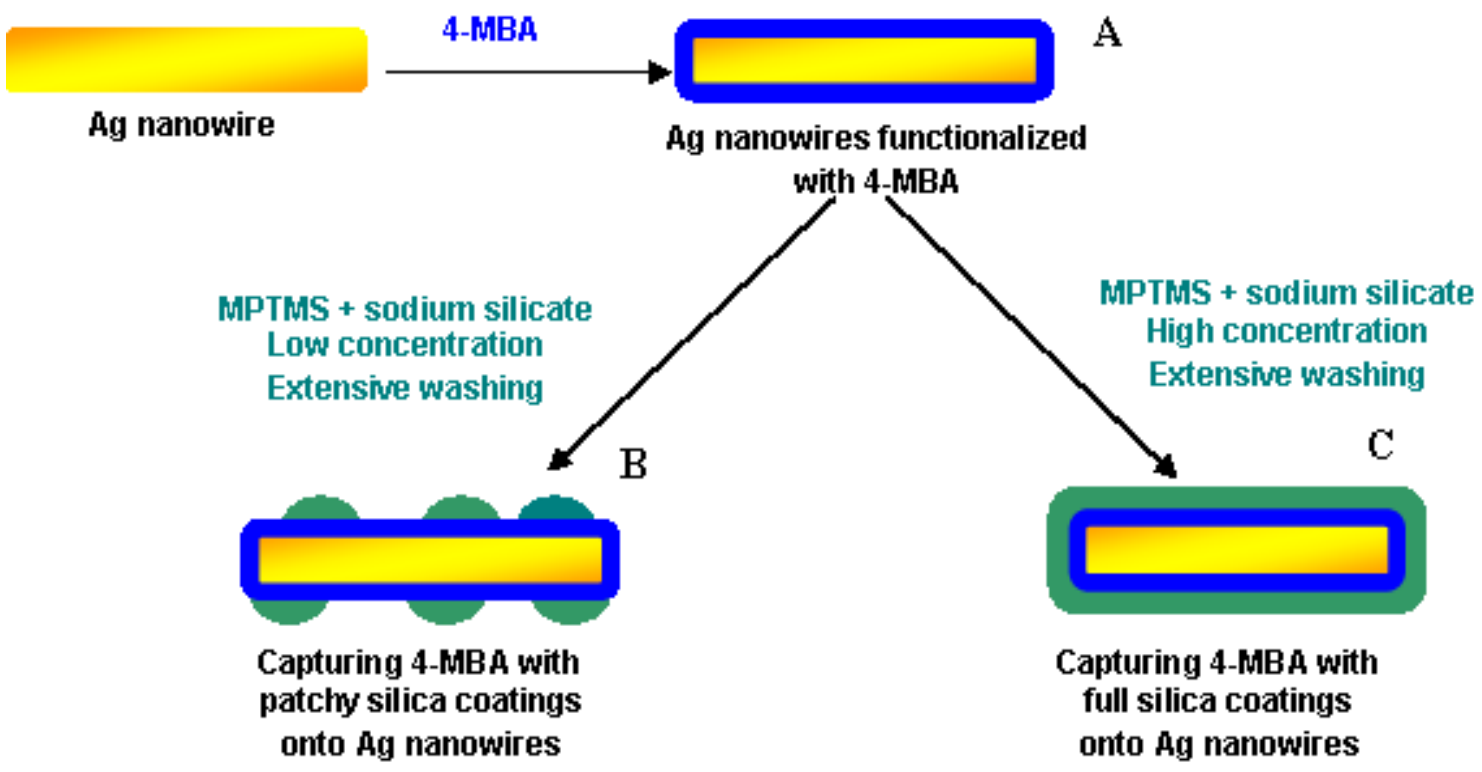

$\begin{array}{ll}\mathrm{HS}-\mathrm{C}_{6} \mathrm{H}_{5}-\mathrm{COOH} & (4-\mathrm{MBA}) \\ \mathrm{HS}\left(\mathrm{CH}_{2}\right)_{3} \mathrm{Si}\left(\mathrm{OCH}_{3}\right)_{3} & \text { (MPTMS) }\end{array}$

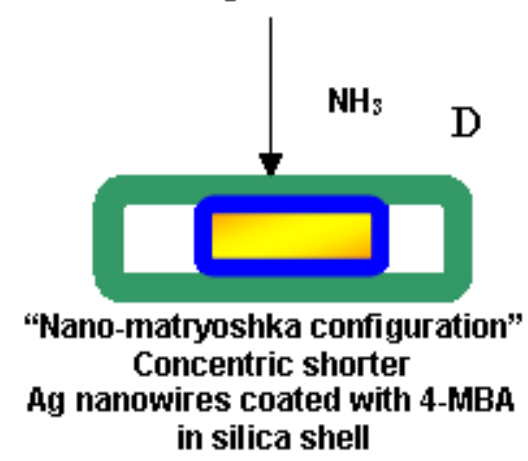

Scheme 1. Cartoon synthesis steps for the preparation of different surface-enhaced Raman substrates. Substrate A: Silver nanowires functionalized with the analyte 4mercaptobenzoic acid (4-MBA); Substrate B: Capturing 4-MBA with patchy silica coats onto silver nanowires; Substrate C: Capturing 4-MBA with full silica coats onto silver nanowires; Substrate D: "Nano-Matryoshka" configuration (Concentric shorter Ag nanowires functionalized with 4-MBA and "oversized" silica shell). 


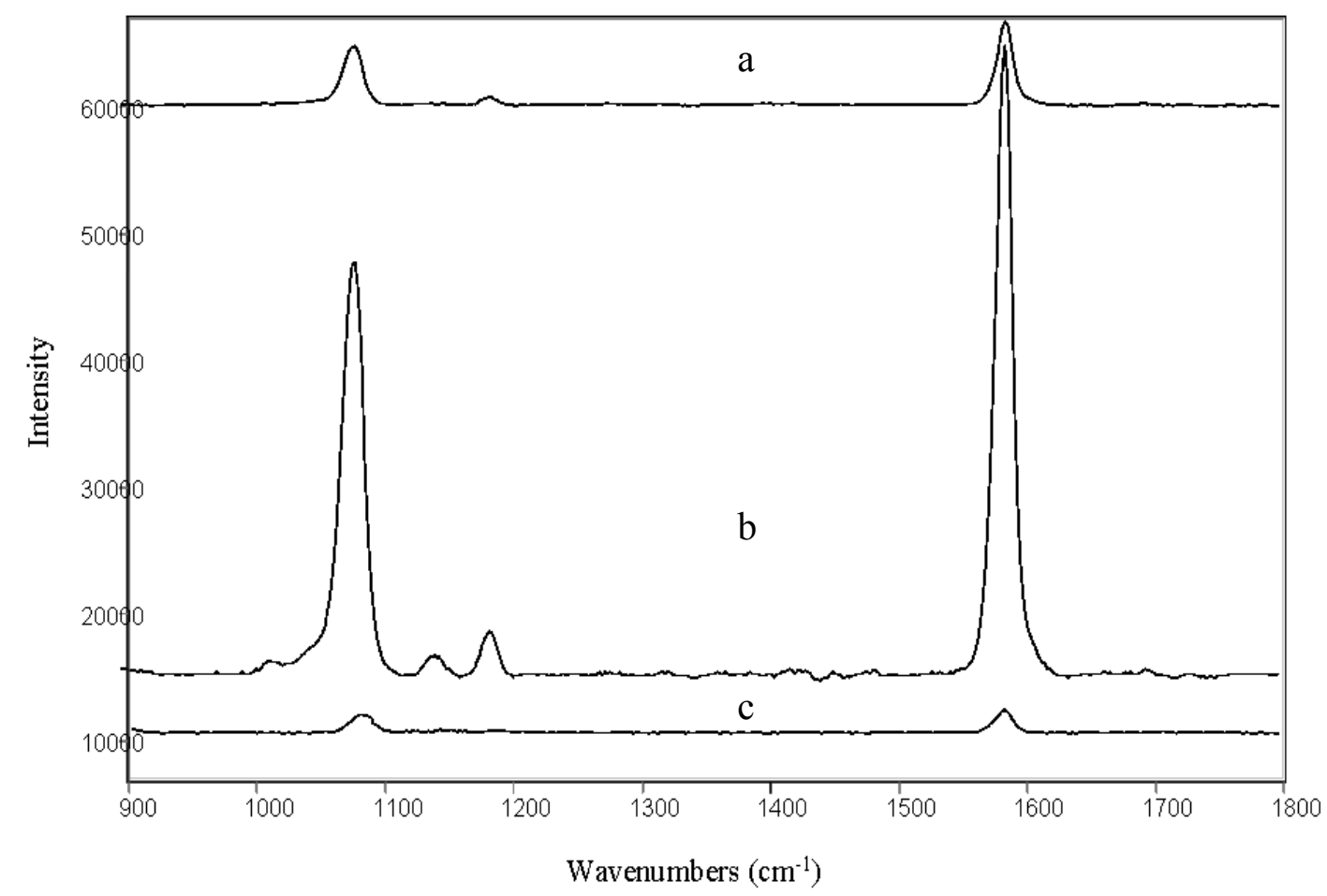

Figure 3. Surface enhanced Raman spectra of 4-MBA using (a) silver nanowires, (b) silver nanowires coated with patchy silica and (c) silver nanowires coated with a full silica coat. The Ag nanowires and nominal 4-MBA concentrations was kept identical in all experiments. Acquisition time: $50 \mathrm{sec}$.

\subsection{Raman Scattering Effects as Function of 4-MBA Concentration on Patchy Silica Coats (Scheme 1, Substrate B).}

We evaluated the SERS activity of the Ag engineered nanowires with patchy silica, while capturing 4-MBA (Scheme 1, Substrate B) as function of 4-MBA concentration. For an accurate comparison, the concentrations of the reactants used for silica deposition were kept constant. This allows for direct comparison of the Raman spectra intensities from probes with different 4-MBA concentrations. (Figure 4 and Figure 5; please note different acquisition time in Figure 4 and 5). As expected, when the concentration of 4-MBA decreased, the intensity of the peaks decreased. However, even when the concentration of analyte decreases to $\mathrm{nM}$ values, the SERS signal was still easily detected, especially for the peak at $1070 \mathrm{~cm}^{-1}$, for an acquisition time of 300 seconds (Figure 5f). 


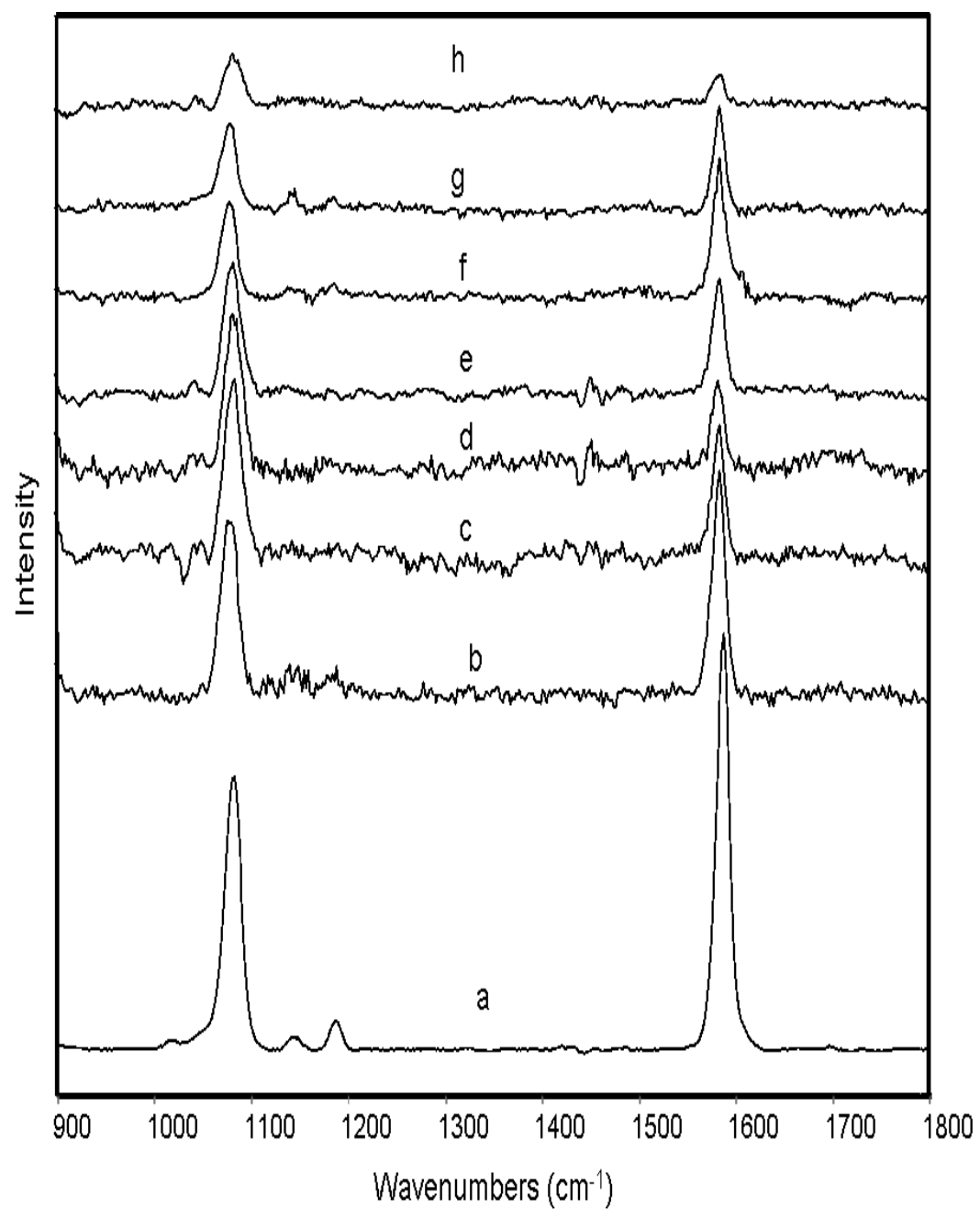

Figure 4. Surface enhanced Raman spectra of 4-MBA using silver nanowires engineered with patchy silica and various bulk concentrations of 4-MBA (a) $650 \mu \mathrm{M}$, (b) $260 \mu \mathrm{M}$, (c) $130 \mu \mathrm{M}$, (d) $65 \mu \mathrm{M}$, (e) $13 \mu \mathrm{M}$, (f) $6.5 \mu \mathrm{M}$, (g) $1.3 \mu \mathrm{M}$, (h) $0.66 \mu \mathrm{M}$. The incubation time in 4-MBA was 24 hrs. Acquisition time was set at 50sec. The Ag nanowire concentration was identical in all experiments. 

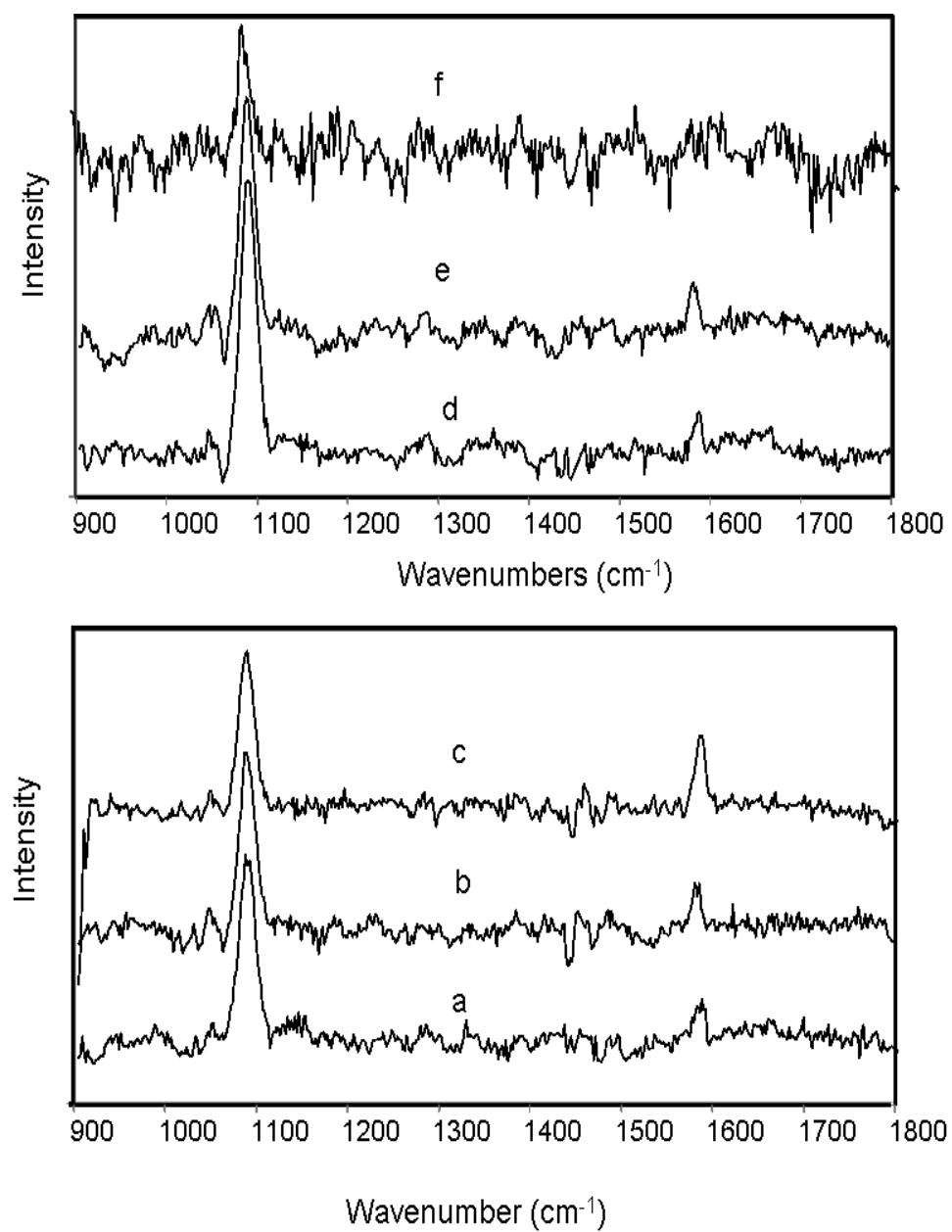

Figure 5. Surface enhanced Raman spectra of 4-MBA using silver nanowires engineered with patchy silica and various bulk solution concentrations of 4-MBA (a) $0.33 \mu M$, (b) $0.16 \mu \mathrm{M}$, (c) $0.08 \mu \mathrm{M}$, (d) $21 \mathrm{nM}$, (e) $10 \mathrm{nM}$, (f) $5 \mathrm{nM}$. The incubation time in 4-MBA was $24 \mathrm{hrs}$. Acquisition time was set at $200 \mathrm{sec}$ for $a, b, c$ and $300 \mathrm{sec}$ for $d, e, f$.

While a non-linear dependence is common in SERS ${ }^{3 a, 4 a}$ surprisingly, in our case, when normalized at $1 \mathrm{sec}$, we can provide a calibration curve for a range of concentration from $260-0.08 \mu \mathrm{M}$ (Figure 6). The calibration curve was obtained based on the vibration spectra of 4-MBA ring-breathing mode set at $1070 \mathrm{~cm}^{-1}$.

The ability to map out a linear dependence of the SERS intensity implies that Ag nanowires engineered with silica deposits can be used as individual SERS nano-sensors to probe diverse environments (Jackson et al 2004). 

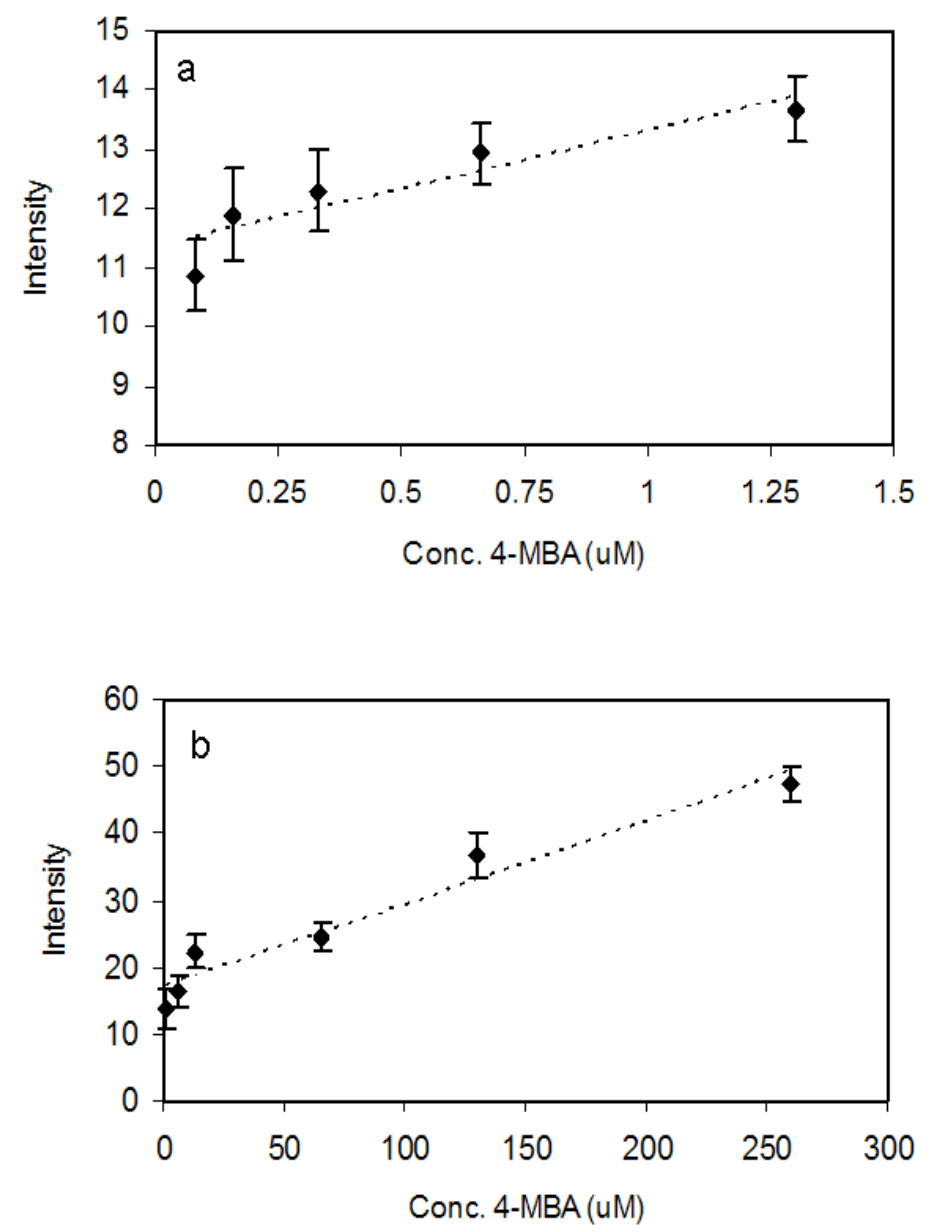

Figure 6. Calibration curve for surface enhanced Raman spectra of 4-MBA using silver nanowires engineered with patchy silica coats by capturing various concentrations of 4$M B A$ (a) from 260-1.3 $\mu M$ 4-MBA and (b) from 1.3-0.08 $\mu M$ 4-MBA.

\subsection{Quantitation of "Hot Spots"}

In order to explore the influence of "hot spots" on the surface Raman scattering experiments, we decided to engineer the presence/absence of an electromagnetic field at the tips of the nanoparticle while encapsulated in full silica shell. So, while encapsulated in the full silica shell, we created supposed "hot spots" on the nanowire surface by an ammonia treatment, based on a procedure reported earlier (Hunyadi et al 2006; Hunyadi Murph et al 2012). The silver nanowires engineered with full silica coats, while capturing 4-MBA, were immersed in highly concentrated ammonia for 20 minutes, while stirring. 
After 24 hrs. immersion time in ammonia, the samples were purified by centrifugation and redispersed in DI water.

The transmission electron micrograph (Figure 7) showed that the ammonia apparently started reacting at the end of the Ag nanowires and produced $\sim 10 \%$ shorter Ag nanowires encapsulated in silica shell (Scheme 1, Substrate D). The Raman spectra recorded on this substrate showed surprisingly enhanced peak intensities which suggest that the tips of the nanowires are indeed "hot spots". However, even if the tips of the wire are "hot spots" and create an enhanced Raman effect, the enhancement effect can't be solely attributed to the tip of the wires but more likely as a contribution of effects, electromagnetic and charge transfer. Experiments are currently in progress to further study the effect of the "hot spots" in the SERS experiments and will be reported at a later date.
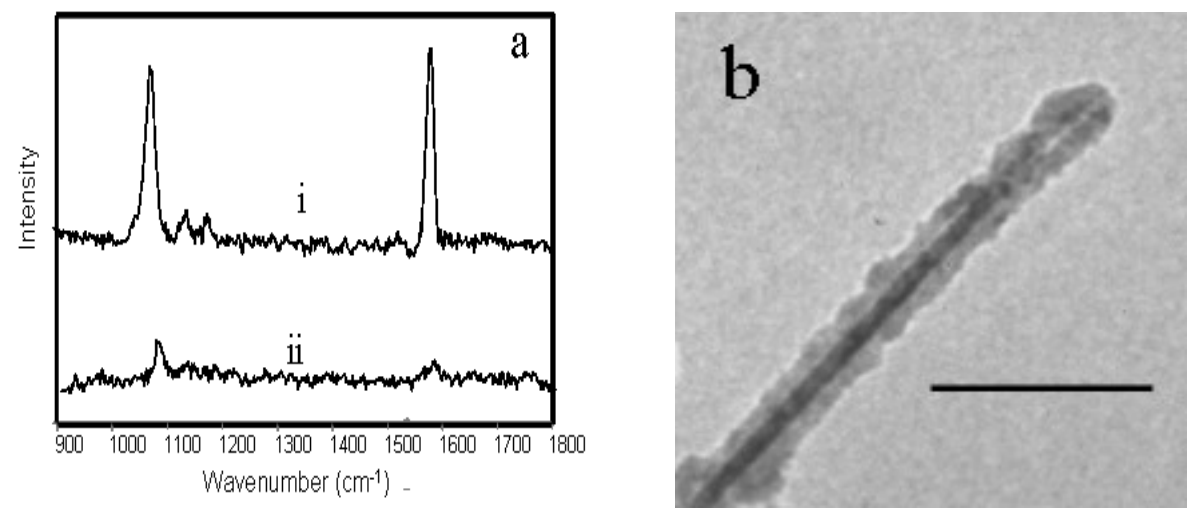

Figure 7. (a) Surface enhanced Raman spectra of $260 \mu M$ 4-MBA captured on silver nanowires engineered with silica coats produced by $25 \mu \mathrm{L}$ of $0.5 \mathrm{mM}$ MPTMS (i) after ammonia treatment: Substrate D and (ii) before ammonia treatment: Substrate C. The results were recorded after 24hrs. incubation time in $260 \mu M$ 4-MBA. Acquisition time: 200 seconds. (b) Transmission electron micrograph of Ag nanowire engineered with silica coats produced by $25 \mu \mathrm{L}$ of $0.5 \mathrm{mM} M P T M S$ and $5 \mu \mathrm{L}$ of $0.252 \mathrm{M} \mathrm{Na}_{2} \mathrm{O}\left(\mathrm{SiO}_{2}\right)_{3}$ while capturing $260 \mu M$ 4-MBA, after ammonia treatment (Substrate D). Scale bar: 100 $n m$.

\section{Discussions}

\subsection{Insights into potential growth mechanism of patchy silica-coated silver nanowires}

In our previous studies, we were able to coat the silver nanowires with a tunable silica shell through an indirect coating approach (Hunyadi et al 2006). Basically, silver 
nanowires prepared by a seedless, surfactantless (Caswell et al 2003) approach were coated with a silica shell through an indirect coating method that involved the use of a bifunctional linker, 3-mercaptopropyltrimethoxysilane $\left(\mathrm{HS}\left(\mathrm{CH}_{2}\right)_{3} \mathrm{Si}\left(\mathrm{OCH}_{3}\right)_{3}\right.$, MPTMS), which binds to the $\mathrm{Ag}$ surface via the thiol group. A thin $\mathrm{SiO}_{2}$ layer is subsequently grown on the MPTMS layer through a modified Stober method.

Subsequently, these core-shell nanowires were exposed in an ammonia environment, which surprisingly produced $\mathrm{Ag}-\mathrm{SiO}_{2}$ nano-peapod architectures. We speculated at that time (Hunyadi et al 2006) that the production of nanopeapod architectures is the result of the periodic steps, defects or molecular-scale "pinholes" present on the silver surface. We assumed that these variances may be more susceptible and reactive to the environment. These speculations were later confirmed while conducting scanning tunneling microscopy studies (Tao et al 2007) on clean silver nanowires. Our studies revealed that the Ag nanowires have a faceted structure with a high density of steps at the facet boundaries. Moreover, monolayer heights have measurable temporal fluctuations at room temperature. Measurement of the step time correlation functions shows that the steps fluctuate via periphery diffusion, with a hopping time constant of 0.10-0.24s (Tao et al 2007, Hunyadi Murph et al 2012). The plastic deformation of these silver nanowires was also studied by AFM nanoindentation and atomistic simulations. AFM images of the residual indent reveal the formation of a neck and surface atomic steps (Lucas et al 2008).

Zeta potential studies conducted here indicated that Ag nanowires have a negative surface charge. This may be due to either citrate or $\mathrm{OH}^{-}$ions present of the $\mathrm{Ag}$ surface (Murphy et al 2005, Hunyadi Murph et al 2011). The presence of these "surfactants" stabilizes the nanowires, due to the electrostatic repulsion, and circumvents nanoparticle's aggregation.

Nevertheless, these "surfactants" can be replaced by ligands binding stronger to the particle surface, such as thiols or amine (Sperling et al 2010, Lin et al 2004, Murphy et al 2005). Thiol groups are particularly of interests since they have the highest binding affinity to noble metal surfaces (approx. $200 \mathrm{~kJ}$ mol-1) (Love et al 2005).

Based on these studies, it is anticipated that incubation of the Ag nanowires into thiols of interests, 4-MBA and MPTMS, is striping off the "surfactants", e.g. citrate, $\mathrm{OH}^{\text {". }}$ 
This will facilitate thiol's chemisorption/binding onto the Ag surface. Since the surface of the Ag nanoparticle consists of not only different crystal facets, but also a large part of edges, steps, pits, terraces and vertices different binding event sites can occur. One can also take into account that thiol molecules have distinctive affinities to different crystal facets and edges or steps (Hostetler et al. 1999). In addition, "bound" thiol ligands appear to be mobile on the surface and able to diffuse to some extent on the particle surface after having bound (Hostetler et al. 1999).

Silver surface are often prone to oxidation (Sur et al 2012). For example, upon oxidation, the adsorptive properties of the silver surface and the orientation of the molecules on the surface change.

The kinetics of self-assembled monolayer formation depends on the adsorbate, solvent substrate properties as well as preparations conditions. There is evidence that self-assembled monolayer formation occurs in two steps, an initial fast step of adsorption and a second slower step of monolayer organization (Schwartz DK 2001). The attractive interactions between the adsorbate and surface are primarily van der Waals forces, electrostatic forces, or combinations of the two (Love et al 2005).

The assembly process begins with a small group of molecules getting close enough that the Van der Waals forces overcome the surrounding force. The forces between the molecules orient them so they are in their straight, optimal, configuration. Then as other molecules come close by they interact with these already organized molecules in the same fashion and become a part of the conformed group. When this occurs across a large area the molecules support each other into forming their selfassembled monolayer shape. Similar to nucleation in metals these thiol islands grow larger they intersect forming boundaries until they end up in the final configuration (Love et al 2005).

While the complete mechanism of the patchy silica-coated silver nanowires is not completely understood we propose that preparation procedure follows the same path. It is certainly possible that those two different thiols involved in the reaction procedure, have different binding affinity onto the Ag nanowire surface. For example, if the 4-MBA is more susceptible to binding to specific areas, such as edges or steps, then these small 
areas of the Ag nanoparticle's surface may be unavailable to the upcoming secondary thiol, MPTMS.

At high concentrations the relative proportion of each modifier on the surface is determined by their relative binding affinities. However, at total modifier concentrations that are closer to those required for monolayer coverage, the equilibrium position resulting from the competitive adsorption will be perturbed because surface binding of the modifier with the highest affinity will deplete its concentration in the bulk phase, reducing its ability to compete for the remaining sites (Stewart et al 2012).

One must take into account that the structure of the self-assembled thiol monolayers is also dependent on the chain length and the structure of both the adsorbate and the substrate. Steric hindrance and metal substrate properties, for example, can affect the packing density of the film (Love et al 2005, Vos et al 2003) while chain length affects self-assembled monolayer thickness (Kaifer 2001). MPTMS has a longer chained while the 4-MBA is shorter and has a benzene group that may hinder the optimal occupancy of the Ag surface. The competing thiols may form patchy thiol islands on the Ag nanowire surface. Addition of sodium silicate in the next step promotes formation of the silica patches on the MPTMS islands. However, it is unclear if the 4-MBA is captured or removed (or both) by patchy silica. A further detailed investigation is being carried out in the laboratory at the present time and will be reported at a later date.

\subsection{SERS studies}

Surface enhanced Raman scattering spectra of 4-mercaptobenzoic acid (4-MBA) self-assembled monolayers (SAMs) with gold nanoparticles of various shapes were reported by our group (Hunyadi et al 2006; Orrendorf et al 2005). ${ }^{6}$ The results showed that by using various gold nanoparticles the SERS enhancement factor is increased $10^{7}$ $10^{9}$ relative to $0.01 \mathrm{M} 4-\mathrm{MBA}$ in aqueous solution and is highly dependent on the nanoparticle shape. We suggested that SERS of the 4-MBA SAMs in this sandwich geometry originates from plasmon coupling between localized surface plasmon of the nanoparticles and surface plasmon of the gold substrate, creating a large localized electromagnetic field enhancement, or SERS "hot spot," for the 4-MBA molecules between the nanoparticles and the planar substrate (Orrendorf et al 2005). 
In addition, we reported earlier surface-enhanced Raman scattering experiments in colloidal solution for 4-mercaptobenzoic on $\mathrm{Ag}$ nanowires, $\mathrm{Ag}-\mathrm{Au}$ bimetallic nanowires and Au nanotubes (Hunyadi et al 2006; Hunyadi Murph et al 2012). In the case of silver nanowires (Scheme 1, Substrate A) we calculated a 6-order of magnitude enhancement, which is surprising since the transverse longitudinal plasmon band is at $400 \mathrm{~nm}$, the longitudinal one is past $1000 \mathrm{~nm}$, which do not overlap with our laser wavelength of $633 \mathrm{~nm}$.

In our case, the results in which patchy coats of silica on silver nanowires produce by far the largest signal for 4-MBA, when compared with "bare" silver or Ag nanowires full coated with silica are really intriguing and may result from several effects. Theoretical analysis indicates that the electrochemical potential may affect the absorption spectrum and surface-enhanced Raman scattering experiment (Blatchford et al 1983). This may occur because of adsorption-desorption of the molecules, formation and dissolution of characteristic surface features, and oxidation-reduction of the molecules or other specific chemical interactions at the electrode. In addition, there will be a redistribution of conduction electrons upon modulation of the surface potential which will be manifested as an altered dielectric constant in the surface region which in turn affects the optical properties (Blatchford et al 1983).

It is well known that the surface dielectric constant depends on the surface morphology. Schatz presented a model for determining the enhancement of local electromagnetic fields which occurs close to metal surfaces through surface plasmon excitation. This model considers a random distribution of metal hemispheroids on a perfectly conducting flat plane. He concluded that surface roughness contributes $10^{2}$ to $10^{3}$ to the overall enhancement with a frequency dependence which is close to that seen experimentally (Laor et al 1982).

Some studies reported that surface plasmons are more efficiently excited in the nanoparticles than the nanorods because of the greater degree of confinement in the former (Schierhorn et al 2006). In the same time, the silica coat shifts the surface resonance condition, which may affect the SERS intensity. This might be the case for our substrate since we produced an array of conductive-insulator silver nano-island-patchy silica architectures. 
According to El-Sayed's group (Mahmoud et al 2012), when the dielectric medium around a nanoparticle is changed anisotropically, either by placing the particle on a substrate or by coating it asymmetrically with a solvent, the plasmon field is distorted, and the plasmonic absorption and scattering spectra could shift differently. This changes the extinction bandwidth of these overlapping absorption and scattering bands.

The enhancement effects in the Raman scattering experiments can be attributed to the electromagnetic theory (Moskovits et al 1984), in which the vibrations along the direction perpendicular to the surface should be more enhanced than the parallel ones. As a result, the in-plane vibration modes of molecules adsorbed in a perpendicular orientation is supposed to be more enhanced than the out-of-plane ones. We assume that, if multilayers of 4-MBA are captured with silica coats, the highly ordered architectures might produce additional vibrations along the direction perpendicular to the surface.

Surface enhancement factors (EF) were calculated by using the following expression (Hunyadi et al 2006; Orendorff et al 2005, 2006):

$$
\mathrm{EF}=\left[\mathrm{I}_{\mathrm{SERS}}\right] /\left[\mathrm{I}_{\text {Raman }}\right] \mathrm{x}\left[\mathrm{M}_{\text {bulk }}\right] /\left[\mathrm{M}_{\mathrm{ads}}\right] \quad \text { Eq. } 1
$$

where $M_{\text {bulk }}$ is the number of molecules sampled in the bulk, $M_{a d s}$ is the number of molecules adsorbed and sampled on the SERS-active substrate, $I_{\text {SERS }}$ is the intensity of a vibrational mode in the surface-enhanced spectrum, and $\mathrm{I}_{\text {Raman }}$ is the intensity of the same mode in the Raman spectrum. We used in our calculations the intensity of $v(\mathrm{CC})_{\text {ring }}$ ringbreathing mode at $1070 \mathrm{~cm}^{-1}$ to identify the enhancement factors. All spectra were normalized for the acquisition time. Raman spectra of solid 4-MBA was used in our calculation enhancement factor for the "bulk" values.

Table 1 shows the enhancement factor calculated when 4-MBA is captured under patchy silica and when 4-MBA is present on the entire silver nanowire surface. 
Table 1. Calculated enhancement factors for different 4-MBA concentration and segment covered with patchy silica. The calculated enhancement factors were calculated when we assumed that 4-MBA was captures by patchy silica coats and when we assume that 4$M B A$ is present on the entire silver surface.

\begin{tabular}{|c|c|c|c|}
\hline $\begin{array}{c}\text { 4-MBA } \\
\text { Concentration } \\
(\mu \mathrm{M})\end{array}$ & $\begin{array}{c}\text { Segment Covered } \\
\text { by Patchy Silica } \\
\text { onto Silver } \\
\text { Nanowires } \\
\text { Surface (\%) }\end{array}$ & $\begin{array}{l}\text { (i) Calculated } \\
\text { Enhancement } \\
\text { Factor: } \\
\text { Captured by } \\
\text { Patchy Silica }\end{array}$ & $\begin{array}{l}\text { (ii) Calculated } \\
\text { Enhancement Factor: } \\
\text { Full Coverage of Silver } \\
\text { Nanowire Surface }\end{array}$ \\
\hline 650 & 0 & $1.1 \pm 0.1 \times 10^{6}$ & $1.1 \pm 0.1 \times 10^{6}$ \\
\hline 650 & $27 \pm 5$ & $6.5 \pm 0.2 \times 10^{8}$ & $1.2 \pm 0.3 \times 10^{8}$ \\
\hline 260 & $29 \pm 7$ & $3.4 \pm 0.2 \times 10^{8}$ & $7.7 \pm 0.2 \times 10^{7}$ \\
\hline 130 & $31 \pm 7$ & $3.1 \pm 0.3 \times 10^{8}$ & $7.5 \pm 0.2 \times 10^{7}$ \\
\hline 65 & $33 \pm 9$ & $2.7 \pm 0.2 \times 10^{8}$ & $6.8 \pm 0.2 \times 10^{7}$ \\
\hline 6.5 & $52 \pm 4$ & $1.0 \pm 0.2 \times 10^{8}$ & $4.1 \pm 0.2 \times 10^{7}$ \\
\hline 0.66 & $50 \pm 6$ & $6.0 \pm 0.5 \times 10^{7}$ & $2.3 \pm 0.4 \times 10^{7}$ \\
\hline 0.166 & $54 \pm 7$ & $5.5 \pm 0.4 \times 10^{6}$ & $2.3 \pm 0.3 \times 10^{6}$ \\
\hline 0.083 & $59 \pm 5$ & $3.5 \pm 0.3 \times 10^{6}$ & $1.8 \pm 0.2 \times 10^{6}$ \\
\hline 0.005 & $61 \pm 6$ & $3.3 \pm 0.5 \times 10^{6}$ & $1.5 \pm 0.4 \times 10^{6}$ \\
\hline
\end{tabular}


There are different assumptions that were taken into account while calculating the enhancement factor. First, the sporadic deposition of the silica along the Ag nanowires requires taking into account the surface area covered by patchy silica that is actually capturing 4-MBA. It is unclear if the 4-MBA is captured or removed (or both) by patchy silica. So, we calculated the enhancement factor with the assumption that: (i) 4MBA is captured by 4-MBA and (ii) 4-MBA is present on the whole surface area of silver nanowire, even outside of the patchy silica coats (Table 1). The segment (length) covered by patchy silica was estimated by the ratio between the distances covered by silica patches and the estimated length of the silver nanowires.

It has been reported earlier that the tip of metallic tip can be considered as a local electromagnetic source (Billot et al 206) which create an enhancement effect in SERS. Recent studies reported by Dawson et al shows that different types of "hot-spot" underpinning the SERS analysis on arrays of vertically aligned multiwalled carbon nanotubes dressed with highly granular Ag. Those at the outer edges of the Ag grains are antenna driven with field enhancement amplified in antenna antinodes while intergrain hotspots are largely independent of antenna activity. Hot-spots between the tops of antennae leaning towards each other also appear to benefit from antenna amplification (Dawson et al 2011).

In our experiments, when $650 \mu \mathrm{M} 4-\mathrm{MBA}$ was captured by $27 \pm 2 \%$ of patchy silica we determined that just $14 \%$ of the tips of the wires are actually both coated with the silica. This is an indication that the more than $65 \%$ of the ends of the wire are open and can create an increased electromagnetic field at the tip of the wire.

Interestingly enough, when silver nanowires, patchy silica-coated silver nanowires or fully coated silver-silica core-shell substrates were used for the same amount of 4-MBA $(650 \mu \mathrm{M})$, significant differences were calculated for the enhancement factor (Table 2). 
Table 2. Calculated enhancement effect vs. \% silica coatings on silver nanowires, patchy silica-coated silver nanowires, and full coated silver-silica core-shell substrates for the same amount of 4-MBA.

\begin{tabular}{|c|c|c|}
\hline Substrate & \% Silica Coating & $\begin{array}{c}\text { Calculated Enhancement } \\
\text { Effect on 650 M 4-MBA }\end{array}$ \\
\hline $\begin{array}{c}\text { Ag nanowires } \\
\begin{array}{c}\text { Patchy Silica Coated Ag } \\
\text { Nanowires }\end{array}\end{array}$ & 0 & $1.10 \pm 0.1 \times 10^{6}$ \\
\hline $\begin{array}{c}\text { Full Coated Silver-Silica } \\
\text { Core-Shell }\end{array}$ & $27 \pm 6$ & $6.50 \pm 0.4 \times 10^{8}$ \\
\hline
\end{tabular}

We presume that a combination of effects is responsible for these enhancement scattering experiments. In agreement with the electromagnetic enhancement mechanism we can imply that the surface enhancement Raman scattering is due to the presence of analyte of interest not just on the side but also, at the tip of the wire; in this way, the end of the nanowires can be considered as "hot spots" and provide an increased electromagnetic field. At the same time, the SERS response is site dependent. Schatz performed calculations and confirm that the local fields are larger on curved regions than flat surfaces (Laor et al 1982). Chemical contributions that can affect SERS response depend of the distinctive surface structure and/or redistribution of conduction electrons which will be manifested as an altered dielectric constant in the surface region are possible explanation to unusual enhancement effects. Theoretical and experimental work published earlier demonstrated the influence of fine geometric nanostructures on local field enhancement which is capable of improving the enhancement factor and better understanding of subtle physical mechanism of SERS (Dawson et al 2011; Tian et al 2011; Wei et al 2008). For example, corrugated nanowires, or nanoparticle-attached corrugated nanowires, influence the enhancement factor (EF) of SERS relative to smooth nanowires. Both the experimental and simulation results show that relatively dense overlapped nanowire networks exhibit a high reproducibility and SERS enhancement 
owing to the formation of homogenously distributed hot spots and the interwire effect (Tian et al 2011).

In any case, Ag nanowires engineered with patchy non-uniform deposits of silica can be used as efficient SERS substrate. Moreover, the versatile chemistry presented by silica coats can be further explored in molecular sensing experiments.

\section{Conclusion}

The new patchy coats of silica on silver nanowires substrate can be used for nanomolar concentration detection of 4-MBA used as analyte of interest. Measurements performed with 4-MBA show a linear calibration graph from $260-0.08 \mu \mathrm{M} 4-\mathrm{MBA}$. Silver nanowires "hot spots" encapsulated in full silica shell was evaluated through an ammonia treatment and showed a $\sim 65 \%$ increased in Raman intensity.

Moreover, the syntheses procedures are performed in aqueous solutions and are amenable to scaling up. While we did not investigate other analytes, we believe that this approach can be tailored to numerous other analytes of interests. Careful selection of the nature of the binding agents could open new avenues toward creation of multi-functional nanostructures with tunable surface properties and chemical specificity. The number and surface functional groups is endless: thiols, amine, silica, titania, DNA, proteins, etc. Our study provides solid evidence that chemical tailoring could be further exploited for production of ordered patchy nanosurfaces. Patchy nanostructures could be further exploited to develop multifunctional nano-surfaces for sensing, labeling and imaging, biological and environmental applications.

\section{References}

Billot L, Chapelle ML, Grimault AS et al (2006) Surface enhanced Raman scattering on gold nanowire arrays: Evidence of strong multipolar surface plasmon resonance enhancement. Chem. Phys. Lett. 422:303-307. 
Blatchford CG, Campbell JR, Creighton JA (1982) Plasma resonance enhanced Raman scattering by adsorbates on gold colloids- The effects of aggregation. Surf. Sci. 120:435-438.

Blatchford CG, Silman O, Kerker M (1983) Potential dependence of Surface- Enhanced Raman Scattering from citrate on colloidal silver. J. Phys. Chem. 87:2503-2508.

Billot L, Chapelle ML, Grimault AS et al (2006) Surface Enhanced Raman Scattering on Gold Nanowire Arrays: Evidence of Strong Multipolar Surface Plasmon Resonance Enhancement. Chem. Phys. Lett. 422:303-307.

Caswell KK, Bender CM, Murphy CJ (2003) Seedless, Surfactantless Wet Chemical Synthesis of Silver Nanowires. Nano Lett. 3:667-669.

Chen MM, Ming M, Katz A (2002) Synthesis and Characterization of Gold-Silica Nanoparticles Incorporating a Mercaptosilane Core-Shell Interface. Langmuir 8:8566-8572.

Creighton JA, Blatchford CG, Albrecht MG (1979) Plasma Resonance Enhancement of Raman-Scattering by Pyridine Adsorbed on Silver of Gold Sol Particles of Size Comparable to the Excitation Wavelength. J. Chem. Soc. Faraday Trans. 75:790798.

Dawson P, Duenas JA, Boyle MG et al. (2011) Combined Antenna and Localized Plasmon Resonance in Raman Scattering from Random Arrays of Silver-Coated, Vertically Aligned Multiwalled Carbon Nanotubes. Nano Lett., 11:365-371.

Doering WE, Nie SM (2002) Single-Molecule and Single-Nanoparticle SERS: Examining the Roles of Surface Active Sites and Chemical Enhancement. J. Phys. Chem. B 106:311-317.

dos Santos DS, Alvarez-Puebla RA, Oliveira ON, Aroca RF (2005) Controlling the Size and Shape of Gold Nanoparticles in Fulvic Acid Colloidal Solutions and Their Optical Characterization using SERS. J. Mater. Chem. 15:3045-3049.

Feldheim D, Foss CA Jr. (2002) Editors, Metal Nanoparticles: Synthesis, Characterization, and Applications, Marcel Dekker Inc., New York.

Imura K, Nagahara T, Okamoto H (2004) Plasmon Mode Imaging of Single Gold Nanorods. J. Am. Chem. Soc. 126:12730-12731. 
Jackson JB, Halas NJ (2004) Surface-enhanced Raman scattering on tunable plasmonic nanoparticle substrates. PNAS 101:17930-17935.

Jensen TR, Schatz GC, Van Duyne RP (1999) Nanosphere Lithography: Surface Plasmon Resonance Spectrum of a Periodic Array of Silver Nanoparticles by UltravioletVisible Extinction Spectroscopy and Electrodynamic Modeling. J. Phys. Chem. B, 103:2394-2401.

Jun YW, Choi JS, Cheon J (2006) Shape Control of Semiconductor and Metal Oxide Nanocrystals Through Nonhydrolytic Colloidal Routes. Angew. Chem. Int. Ed. Engl. 45:3414-3439.

Kelly KL,Coronado E, Zhao LL, Schatz GC (2003) The Optical Properties of Metal Nanoparticles: The Influence of Size, Shape, and Dielectric Environment. J. Phys. Chem. B, 107:668-677.

Kerker M, Siiman O, Wang, DS (1984) Effect of Aggregates on Exctinction and SurfaceEnhanced Raman Scattering Spectra of Colloidal Silver. J. Phys. Chem 88:31683170 .

Kneipp K, Kneipp H, Itzkan I, Dasari RR, Feld MS (1999) Ultrasensitive Chemical Analysis by Raman Spectroscopy. Chem. Rev. 99:2957-2975.

Kneipp K, Wang Y, Kneipp H et al (1997) Single Molecule Detection Using SurfaceEnhanced Raman Scattering (SERS). Phys. Rev. Lett. 78:1667-1670.

Kneipp K, Kneipp H, Itzkan I, Dasari RR, Feld MS (1999) Surface-Enhanced Raman Scattering: A New Tool for Biomedical Spectroscopy. Curr. Science 77:915-924.

Laor U, Schatz GC (1982) The effect of randomly distributed surface bumps on local field enhancements in Surface Enhanced Raman Spectroscopy J. Phys. Chem. 76:2888-2899.

Lisiecki I (2005) Size, Shape, and Structural Control of Metallic Nanocrystals. J. Phys. Chem. B. 109:12231-12244.

Liu GL, Lu Y, Kim J, Doll JC, Lee LP (2005) Magnetic Nanocrescents as Controllable Surface-Enhanced Raman Scattering Nanoprobes for Biomolecular Imaging. Adv. Mat. 17:2683-2687.

Hartschuh A, Anderson N, Novotny, L (2003) Near-field Raman Spectroscopy Using a Sharp Metal Tip. J. Microsc. 210:234-240. 
Hayazawa N, Inouye Y, Sekkat Z, Kawata S (2001) Near-field Raman Scattering Enhanced by a Metallized Tip. Chem. Phys. Lett. 335:369-374.

Hostetler MJ, Templeton AC, Murray RW (1999) Dynamics of place-exchange reactions on monolayer-protected gold cluster molecules. Langmuir 15:3782-3789.

Hunyadi S, Murphy C (2006) Bimetallic silver-gold nanowires: fabrication and use in surface-enhanced Raman scattering. J Mater Chem 16:3929-3935

Hunyadi SE, Murphy CJ (2006) Tunable One-Dimensional Silver-Silica Nanopeapod Architectures. J. Phys. Chem. B 110:7226 -7231.

Hunyadi Murph SE, Serkiz S, Fox E et al (2011) Synthesis, functionalization, characterization and application ofcontrolled shape nanoparticles in energy production. Book Chapter ACS Symposium Series, vol 1064, Chapter 8, 127163.

Hunyadi Murph SE., Heroux K, Turick, C et al (2012) Metallic and Hybrid Nanostructures: Fundamentals and Applications in Applications of Nanomaterials. Series ISBN: 1-62699-000-X, Vol.4: Nanomaterials and Nanostructures, Volume (4), J.N. Govil, ISBN: 1-62699-004-2, Studium Press LLC, USA.

Hunyadi Murph SE, Murphy C, Colon-Mercado H et al (2011) Tuning of Size and Shape of Au-Pt Nanocatalyst for Direct Methanol Fuel Cells J. Nanoparticle Research 13:6347-6364.

Kaifer A (2001) Supramolecular Electrochemistry. Coral Gables. Wiley VCH. pp. 191193.

Lin SY, Tsai YT, Chen CC et al (2004) Two-step functionalization of neutral and positively charged thiols onto citrate-stabilized Au nanoparticles. J. Phys. Chem. B 108:2134-2139.

Liz-Marzán LM, Giersig M, Mulvaney P (1996) Synthesis of Nanosized Gold-Silica Core-Shell Particles. Langmuir 12:4329-4335.

Love JC, Estroff LA, Kriebel JK et al (2005) Self-Assembled Monolayers of Thiolates on Metals as a Form of Nanotechnology. Chem. Rev. 105:1103-1170.

Lucas M, Leach AM, McDowell MT, Hunyadi SE, Gall K, Murphy CJ, Riedo E (2008) Plastic Deformation of Pentagonal Silver Nanowires: Comparison Between AFM 
Nanoindentation and Atomistic Simulations. Physical Review B 77:24542012452014.

Mahmoud MA, Chamanzar M, Adibi A, El-Sayed MA (2012) Effect of the Dielectric Constant of the Surrounding Medium and the Substrate on the Surface Plasmon Resonance Spectrum and Sensitivity Factors of Highly Symmetric Systems: Silver Nanocubes. J. Am. Chem. Soc, 134:6434-6442.

McLellan JM, Xiong YJ, Hu M, Xia YN (2006) Surface-Enhanced Raman Scattering of 4-Mercaptopyridine on Thin Films of Nanoscale Pd Cubes, Boxes, and Cages. Chem. Phys. Lett., 417: 230-234.

Mine EM, Samada A, Kobayashi Y et al (2003) Direct coating of gold nanoparticles with silica by a seeded polymerization technique. J. Colloid Interface Sci. 264:385390.

Moskovits M, Sun JS (1984) Surface Selection-Rules for Surface-Enhanced RamanSpectroscopy- Calculations and Application to the Surface-Enhanced Raman Spectrum of Phthalazine on Silver. J. Phys. Chem. 1984, 88, 5526-5530.

Murphy C, Sau T, Hunyadi S et al (2005) Anisotropic metal nanoparticles: synthesis, assembly, and optical applications. J Phys Chem B 109:13857-13870.

Natan MJ., Lyon LA, Keating CD et al (1998) Raman Spectroscopy. Anal. Chem, 70:341R-361R.

Nikoobakht B, El-Sayed MA (2003) Surface-Enhanced Raman Scattering Studies on Aggregated Gold Nanorods J. Phys. Chem. A 107:3372-3378.

Obare SO, Jana NR, Murphy CJ (2001) Preparation of polystyrene- and silica-coated gold nanorods and their use as templates for the synthesis of hollow nanotubes. Nano Lett., 1:601-603.

Orendorff CJ, Gole A, Sau TK, Murphy CJ (2005) Surface-Enhanced Raman Spectroscopy of Self-Assembled Monolayers: Sandwich Architecture and Nanoparticle Shape Dependence. Anal. Chem. 77:3261-3266.

Orendorff CJ, Gearheart L, Jana NR, Murphy CJ (2006) Aspect Ratio Dependence on Surface Enhanced Raman Scattering Using Silver and Gold Nanorod Substrates. Phys. Chem. Chem. Phys. 8:165-170. 
Park HK, Kim K (2006) Facile Method to Prepare Surface-Enhanced-Raman-ScatteringActive Ag Nanostructures on Silica Spheres. Langmuir 8083-8086.

Pinzaru SC, Pavel I, Leopold N, Kiefer W (2004) Identification and Characterization of Pharmaceuticals using Raman and Surface-Enhanced Raman Scattering. J. Raman Scattering 35:338-346.

Puntes VF, Krishnan KM, Alivisatos AP (2001) Colloidal Nanocrystal Shape and Size Control: The Case of Cobalt. Science 291:2115-2117.

Ruan CM, Wang W, Gu AH (2006) Surface-Enhanced Raman Scattering for Perchlorate Detection Using Cystamine-Modified Gold Nanoparticles. Anal. Chem. Acta 567:114-120.

Santra P, Zhang K, Wang R et al. (2001) Conjugation of Biomolecules with Luminophore-Doped Silica Nanoparticles for Photostable Biomarkers. Anal. Chem 73:4988 - 4993.

Schwartz DK (2001) Mechanisms and kinetics of self-assembled monolayer formation. Annu. Rev. Phys. Chem. 52: 107-37.

Schierhorn M, Lee SJ, Boettcher SW et al (2006) Metal-silica hybrid nanostructures for surface-enhanced Raman spectroscopy. Adv. Mater. 18:2829-2832.

Scott RWJ, Wilson OM, Crooks RM (2005) Synthesis, Characterization, and Applications of Dendrimer-Encapsulated Nanoparticles. J. Phys. Chem. B., 109:692-704.

Solecka-Cermakova K. (1996) Structural Characteristics of Ag Colloid-Adsorbate Films Determined from Transmission Electron Microscopic Images: Fractal Dimensions, Particle Size, and Spacing Distributions and Their Relationship to Formation and Optical Responses of the Films. J. Phys. Chem. 100:4954-4960.

Song C, Abell J, He Y, Hunyadi Murph SE et al (2012) Gold-Modified Silver Nanorod Arrays: Growth Dynamics and Improved SERS Properties. J. Mater. Chem. 22:1150-1159.

Sperling RA, Parak WJ (2010) Surface modification, functionalization and bioconjugation of colloidal inorganic nanoparticles Phil. Trans. R. Soc. A 368:1333-1383. 
Stewart I, Zheng S, McCourt MR, Bell SEJ (2012) Controlling Assembly of Mixed Thiol Monolayers on Silver Nanoparticles to Tune Their Surface Properties ACS Nano, 6:3718-3726.

Stöckle RM, Suh YD, Deckert RZV (2000) Nanoscale Chemical Analysis by TipEnhanced Raman Spectroscopy. Chem. Phys. Lett. 318:131-136.

Sur I, Altunbek M, Kahraman M, Culha M (2012) The influence of the surface chemistry of silver nanoparticles on cell death Nanotechnology 23:375102.

Tao CG, Cullen WG, Williams ED, Hunyadi SE, Murphy CJ ( 2007) Surface Morphology and Step Fluctuations on Silver Nanowires.Surface Science, 601:4939- 4943.

Tian C, Ding C, Liu S et al (2011) Nanoparticle Attachment on Silver Corrugated-Wire Nanoantenna for Large Increases of Surface-Enhanced Raman Scattering. ACS Nano, 5:9442-9449.

Vo-Dinh T, Yan F, Wabuyele MB (2006) Surface-Enhanced Raman Scattering for Biomedical Diagnostics and Molecular Imaging. SERS: Phys. Appl. Topics Appl. Phys. 103:409-426.

Vos JG, Forster RJ, Keyes TE (2003). Interfacial Supramolecular Assemblies. Wiley. pp. 88-94.

Wang H, Levin CS, Halas NJ (2005) Nanosphere Arrays with Controlled Sub-10-nm Gaps as Surface-Enhanced Raman Spectroscopy Substrates. J. Am. Chem. Soc. 127:14992-14993.

Wei H, Hao F, Huang YZ et al (2008) Polarization dependence of surface-enhanced Raman scattering in gold nanoparticle-nanowire systems. Nano Lett. 8:24972502.

Wiley B, Sun Y, Xia Y (2005) Polyol Synthesis of Silver Nanostructures: Control of Product Morphology with Fe(II) or Fe(III) Species. Langmuir 21:8077-8080.

Willner I, Shipway AN, Katz E (2000) Nanoparticle Arrays on Surfaces for Electronic, Optical, and Sensor Applications. Chem. Phys. Chem. 1:18-52.

Xia Y, Yang P, Sun Y, Wu Y et al (2003) One Dimensional Nanostructures: Synthesis, Characterization, and Applications. Adv. Mater. 15:353 - 389. 
Yin Y, Alivisatos AP (2005) Colloidal Nanocrystal Synthesis and the Organic-Inorganic Interface. Nature, 437:664-670.

Zhang X, Zhao J, Whitney AV et al (2006) Ultrastable Substrates for Surface-Enhanced Raman Spectroscopy: A12O3 Overlayers Fabricated by Atomic Layer Deposition Yield Improved Anthrax Biomarker Detection. J. Am. Chem. Soc. 128:1030410309. 